\title{
Metabolite profiling and biological activities of bioactive compounds produced by Chrysosporium lobatum strain BK-3 isolated from Kaziranga National Park, Assam, India
}

\author{
C Ganesh Kumar ${ }^{1 *}$, Poornima Mongolla ${ }^{1}$, Pombala Sujitha', Joveeta Joseph ${ }^{1}$, K Suresh Babu², Gangi Suresh², \\ Kallaganti Venkata Siva Ramakrishna ${ }^{3}$, Uppula Purushotham ${ }^{4}$, G Narahari Sastry ${ }^{4}$ and Ahmed Kamal ${ }^{1}$
}

\begin{abstract}
In an ongoing survey for bioactive potential of microorganisms from different biosphere zones of India, a new Chrysosporium lobatum strain BK-3 was isolated from soil sample collected from a biodiversity hotspot, Kaziranga National Park, Assam, India. Bioactivity-guided purification resulted in the isolation of two bioactive compounds whose chemical structures were elucidated by ${ }^{1} \mathrm{H}$ and ${ }^{13} \mathrm{C}$ Nuclear Magnetic Resonance (NMR), 2D-NMR, Fourier Transform Infra-red (FT-IR) and mass spectroscopic techniques, and were identified as $\alpha, \beta$-dehydrocurvularin and curvularin. Only curvularin exhibited 80\% acetylcholinesterase (AChE) inhibitory activity. Detailed ligand receptor binding interactions were studied for curvularin by molecular docking studies. Further, both curvularin and a, $\beta$-dehydrocurvularin had similar level of cytotoxicity against different human tumour cell lines like A549, HeLa, MDA-MB-231 and MCF-7, while a, $\beta$-dehydrocurvularin was active against COLO 205 with a $\mathrm{IC}_{50}$ of $7.9 \mu \mathrm{M}$, but curvularin was inactive. $\alpha$, $\beta$-Dehydrocurvularin also showed good superoxide anion scavenging activity with an $E_{50}$ value of $16.71 \mathrm{mg} \mathrm{ml}^{-1}$. Hence, both these compounds exhibited differences in bioactive profiles and this was probably associated with their minor structural differences. This is a first report on bioactive compounds exhibiting AChE inhibitory, cytotoxicity and antioxidant activities from Chrysosporium lobatum strain BK-3.
\end{abstract}

Keywords: Acetylcholinesterase inhibitors, Chrysosporium lobatum, Curvularin, a, $\beta$-Dehydrocurvularin, Molecular docking

\section{Introduction}

Alzheimer's disease (AD), the most common neurodegenerative disorder associated with ageing, is accompanied by severe deficiency in choline acetyltransferase activity in the hippocampus and cerebral cortex. A significant correlation has been found between the decrease in cortical cholinergic activity and the deterioration of mental test scores in patients suffering from AD. Therefore, potentiation of central cholinergic activity has been proposed as a possible therapeutic approach to improve cognitive function in AD patients (Johnson et al. 2000). Among the most promising strategies is the elevation of

\footnotetext{
* Correspondence: cgkumar5@gmail.com

${ }^{1}$ Chemical Biology Laboratory, CSIR-Indian Institute of Chemical Technology, Uppal Road, Hyderabad 500007, Andhra Pradesh, India

Full list of author information is available at the end of the article
}

the brain concentration of the neurotransmitter acetylcholine by centrally acting acetylcholinesterase (AChE, EC 3.1.1.7) inhibitors. During the past decade, several cholinergic drugs have been launched in the market, including the synthetic compounds like tacrine (Bartus et al. 1982), donepezil (E2020) (Watkins et al. 1994), galanthamine (Davis et al. 1995), and rivastigmine (Kawakami et al. 1996). It has been reported that use of these drugs produce significant cognitive improvement (Nightingale 1997; Kryger et al. 1999; McGleenon et al. 1999). However, a major drawback in the widespread use of these synthetic compounds as a general therapy is the appearance of undesirable side effects such as hepatotoxicity, which imposes severe dose-limits (Watkins et al. 1994).

The comprehensive study of the AChE/inhibitor complexes by X-ray crystallography had indicated that $\mathrm{AChE}$ 
possessed a narrow gorge with two separate ligand binding sites, an acylation site (active site) and a peripheral site which was also called peripheral anionic site. Simultaneous binding to the catalytic anionic subsite and the peripherical anionic site is responsible for the enhanced binding of gorge-spanning ligands, such as galanthamine and donepezil (E2020). Recent biochemical studies have shown that the peripherical anionic site is also implicated in promoting aggregation of the beta-amyloid $(A \beta)$ peptide responsible for the neurodegenerative process in AD (Greenblatt et al. 1999; Tariot 2001; Bar-On et al. 2002). Thus, the design of inhibitors interacting with the peripherical anionic site is of clear potential interest for treatment of AD (Zeng et al. 1999).

Bioprospecting is a term coined recently to refer to the search for novel products or microorganisms of economic importance from the world's biota. Over the past few years a renewed interest has been generated on the investigation of fungi as a potential source of novel bioactive compounds like immunosuppressive, anti-cholesterolemic and anti-tumour agents (Harvey 2000; Strobel 2000; Tulp and Bohlin 2004). Most fungi investigated have been isolated from soil samples and they have provided a broad spectrum of secondary metabolites with diverse chemical structures; the most exciting recent discoveries have come from exploration of fungi surviving in unusual ecological niches like rain forests (Strobel 2000), marine sponges (Höller et al. 2000), and mangroves (Lin et al. 2001). In particular, $\mathrm{AChE}$ inhibitors were reported to be produced by some soil fungi like arisugacin A and B from Penicillium sp. FO 4259 (Kuno et al. 1996), quinolactacins A1 and A2 from Penicillium citrinum (Kim et al. 2001) and terreulactones A, B, C and D from Aspergillus terreus (Kim et al. 2003). The present study reports on the isolation and characterization of fungal acetylcholinesterase (AChE) inhibitors from the hitherto unexplored biosphere, the Kaziranga National Park, Assam, India. The Kaziranga National Park $\left(26^{\circ} 30^{\prime}\right.$ to $26^{\circ} 45^{\prime} \mathrm{N}$ and $93^{\circ} 05^{\prime}$ to $\left.93^{\circ} 40^{\prime} \mathrm{E}\right)$, Assam, India is located on the southern bank of the Brahmaputra river at the foot of the Mikir - Kirbi Anglang Hills and is declared as a World Heritage site by UNESCO in 1985. This park is one of the last areas in Eastern India and is almost undisturbed by human and has a unique natural riverine landscape of sheer forest, tall elephant grass, rugged reeds, marshes and shallow pools inhabited by the world's largest population of one-horned rhinoceroses and diverse range of other wild animals (UNEP 2005; UNESCO 2010). Considering the importance of this hitherto unexplored biosphere, fungi were isolated from different soil and dung samples collected from this forest and the metabolic profiling of secondary metabolites produced by a newly isolated Chrysosporium lobatum strain BK-3 was carried out and assessed for their AChE inhibition, antimicrobial, cytotoxicity and antioxidant activities.

\section{Materials and methods Chemicals used}

Acetylcholinesterase (AChE) from electric eel (EC 3.1.1.7); acetylthiocholine iodide (ATCI); 5, $5^{\prime}$-dithiobis(2-nitrobenzoic acid) (DTNB); Trizma hydrochloride (Tris- $\mathrm{HCl}$ ) buffer solution, $\mathrm{pH} 8.0$ and reference compound like galanthamine hydrobromide from Lycoris sp. were purchased from Sigma (St. Louis, MO, USA).

\section{Fungal strain and fermentation conditions}

The fungal strain BK-3 was isolated from a forest soil sample collected from the Kaziranga National Park, Assam, India, as part of a bioactives screening program (Kumar et al. 2010). The pure culture was maintained in the in-house culture repository of the institute with the accession number ICTF-039 and based on the cultural and morphological characteristics of spore and hyphae by lactophenol blue staining and microscopic observation, the strain BK-3 was identified as Chrysosporium lobatum. The strain BK-3 was pre-cultured aerobically at $30^{\circ} \mathrm{C}$ for 5 days in $1 \mathrm{~L}$ Erlenmeyer flasks containing $250 \mathrm{ml}$ of potato dextrose broth medium and agitated at $150 \mathrm{rev} \mathrm{min}^{-1}$ in an orbital shaker for $120 \mathrm{~h}$. The fermented medium was later filtered through a muslin cheese cloth to remove the fungal biomass and then the infiltrate was centrifuged at $2000 \mathrm{rpm}$ to obtain a cellfree supernatant.

\section{Extraction, analysis and purification of bioactive metabolites}

Bioactive compounds were extracted from the cell-free supernatant by absorption onto Diaion HP-20 (3\%, Supelco, Bellafonte, PA, USA) resin. The resin was washed with water and then extracted with methanol to obtain the crude extract. The crude extract was analyzed by thin-layer chromatography (TLC) on silica gel 60 plates $\left(\mathrm{F}_{254}\right.$, Merck). Plates were developed in a methanol-chloroform (5:95) solvent mixture and visualized under UV light at $254 \mathrm{~nm}$ which revealed the presence of two bioactive compounds. The extracted compound was further concentrated under reduced pressure on a rotary vacuum evaporator (Rotavapor R-205, Büchi, Switzerland) and further profiled on silica gel (60-120 mesh) column $(3 \times 60 \mathrm{~cm})$ with methanol-chloroform solvent system as a mobile phase. Spot 1 was eluted in methanolchloroform mixture $(1: 99, \mathrm{v} / \mathrm{v})$. The same solvent mixture was continued till compound 1 was completely eluted and after drying gave a pale yellow powder. Spot 2 was eluted in methanol-chloroform mixture (1.5:98.5, v/v), and drying of the fractions resulted in compound 2 as white powder. Both compounds were UV-active and visualized under UV light at $254 \mathrm{~nm}$, as well as by spraying with phosphomolybdic acid followed by heating at $100^{\circ} \mathrm{C}$ for 2-3 min which appeared as blue coloured spots. 


\section{Structural characterization}

The purified compounds were further subjected to ${ }^{1} \mathrm{H}$ and ${ }^{13} \mathrm{C}$ NMR, FT-IR, ESI-MS along with 2D-NMR spectroscopic studies like HMBC, HSQC, ${ }^{1} \mathrm{H}_{-}{ }^{1} \mathrm{H}-\mathrm{COSY}$, NOSEY and DEPT-135 studies for elucidating their structures. The $\mathrm{UV}$ and visible spectra were measured by dissolving the samples in spectroscopic acetonitrile and recorded at $30^{\circ} \mathrm{C}$ on a UV-visible double beam spectrophotometer (Lambda 25, Perkin-Elmer, Shelton, CT). Nuclear magnetic resonance (NMR) spectra were recorded on a Bruker Avance $300 \mathrm{MHz}$ NMR spectrometer (Bruker, Switzerland). The ${ }^{1} \mathrm{H}$ and ${ }^{13} \mathrm{C}$ NMR spectra were determined in deuterated chloroform at room temperature, and chemical shifts were represented in $\delta$ values ( $\mathrm{ppm}$ ) with tetramethylsilane (TMS) as an internal standard. The Fourier transform infrared spectrum (FT-IR) was performed using the ThermoNicolet Nexus 670 FT-IR spectrophotometer (Thermo Fisher Scientific Inc., Madison, WI, USA) using $\mathrm{KBr}$ pellets and spectra were collected at a resolution of $4 \mathrm{~cm}^{-1}$ in the wavenumber region of $400-4,000 \mathrm{~cm}^{-1}$. The high resolution mass spectra (HR-MS) were recorded on a QSTAR XL Hybrid ESI-Q TOF mass spectrometer (Applied Biosystems Inc., Fosters City, CA, USA). Electrothermal Digital 9000 Series melting point apparatus (Model IA9200, Barnstead, UK) was used for determining the melting point of the purified compounds. The purified sample (1 mg) of each bioactive compound was placed in a glass capillary tube and this tube was placed in an aluminium heating block which was heated at a fast ramp rate of $10^{\circ} \mathrm{C} \mathrm{min}{ }^{-1}$ from a temperature range of 25 to $300^{\circ} \mathrm{C}$ for melting point measurement.

\section{Antimicrobial activity}

Antimicrobial activity was determined using the broth dilution method as described previously (Kumar and Mamidyala 2011). The target strains used for screening the antimicrobial activities were Micrococcus luteus MTCC 2470, Staphylococcus aureus MTCC 96, Staphylococcus aureus MLS16 MTCC 2940, Bacillus subtilis MTCC 121, Escherichia coli MTCC 739, Pseudomonas aeruginosa MTCC 2453, Klebsiella planticola MTCC 530 and Candida albicans MTCC 3017. These strains were procured from Microbial Type Culture Collection and Gene Bank (CSIR-Institute of Microbial Technology, Chandigarh, India).

\section{In vitro cytotoxicity testing}

Cytotoxicity of the compounds was assessed on the basis of the measurement of the in vitro growth in the 96 well plates by cell-mediated reduction of tetrazolium salt to water insoluble formazan crystals by a previously described method (Mosmann 1983). Cell lines for testing in vitro cytotoxicity included HeLa derived from human cervical cancer cells (ATCC No. CCL-2), A549 derived from human alveolar adenocarcinoma epithelial cells (ATCC No. CCL-185), MDA-MB-231 derived from human breast adenocarcinoma cells (ATCC No. HTB-26), MCF7 derived from human breast adenocarcinoma cells (ATCC No. HTB-22), COLO 205 derived from human colon cancer cell line (ATCC No CCL-222), K562 derived from human chronic myelogenous leukemia cell line (ATCC No. CCL-243) and HEK 293 derived from human embryonic kidney cell line (ATCC No. CRL-1573) were obtained from American Type Culture Collection, Manassas, VA, USA. The $\mathrm{IC}_{50}$ values $(50 \%$ inhibitory concentration) were calculated from the plotted absorbance data for the dose-response curves. The $\mathrm{IC}_{50}$ values (in $\mu \mathrm{M}$ ) were expressed as the average of two independent experiments.

\section{Antioxidant activities}

The purified compounds were investigated for their antioxidant potentials in different oxidizing systems, viz., radical scavenging activity by 1,1-diphenyl-2-picrylhydrazyl (DPPH) reduction (Kumar et al. 2011), superoxide radical scavenging activity (Liu et al. 1997), inhibition of lipid peroxidation (Zhang and Yu 1997) and hemolysis in erythrocyte membrane stabilization (Ng et al. 2000) at different concentrations. Ascorbic acid and luteolin were also employed as standards in these assays. All experiments were carried out in triplicates and the $\mathrm{EC}_{50}$ (50\% effective concentration) values $\left(\mu \mathrm{g} \mathrm{ml}^{-1}\right)$ were expressed as the mean \pm standard deviation (S.D.).

\section{In vitro acetylcholinesterase (AChE) assay}

AChE inhibitory activity for the purified compounds was performed using the modified method of Ellman et al (1961) in 96 well plates (Rhee et al. 2001). The concentration of the compound that inhibited $50 \%$ of $\mathrm{AChE} \mathrm{ac-}$ tivity $\left(\mathrm{IC}_{50}\right)$ was estimated by plotting percent activity and percent inhibition of $\mathrm{AChE}$ versus inhibitor concentrations on the same graph. The concentration at the intersection of these two curves was the $\mathrm{IC}_{50}$ value.

\section{Protein-ligand docking studies}

The molecular docking studies were performed using various docking softwares like GOLD (Genetic Optimization for Ligand Docking, version 3.2) and CDOCKER (in Discovery Studio 2.5) on the curvularin molecule with the well-known complex acetylcholinesterase complexed with E2020 which is marketed as Aricept (PDBID: 1EVE) (Badrinarayan et al. 2011). The Accelrys Discovery studio protein preparation wizard was used to prepare the protein with default settings. The ligands were subjected to energy minimization at AM1 level of theory. The default parameters in GOLD 3.2 such as number of islands 5, population size of 100 , number of operations was 100,000 , a niche size of 2, a selection pressure of 1 , the van der 
Waals and hydrogen bonding were set to 4.0 and 2.5 , respectively, have been used to perform GOLD docking calculations (Srivani et al. 2007; Ravindra et al. 2008; Badrinarayan and Sastry 2011). The CDOCKER had an all-atom CHARMm force field-based docking algorithm and used the soft-core potentials with an optional grid representation to dock ligands into the active site of the receptor. CDOCKER generated the random ligand conformations through molecular dynamics simulation. CDOCKER docking was done with the default parameters using the ligand E2020 as the center.

\section{Results}

Purification, characterization and structure elucidation of bioactive compounds

The culture filtrate of strain BK-3 revealed the presence of two major spots in TLC with $R f$ values of 0.5 for compound 1 and 0.3 for compound 2 which were separated in a solvent mixture of methanol:chloroform solvent system. Compound 1: Yield: $50 \mathrm{mg} \mathrm{l}^{-1}$; pale yellow powder; heat stable and showed no change in its activity, when autoclaved at $121^{\circ} \mathrm{C}$ for $30 \mathrm{~min}$; mp $218-220^{\circ} \mathrm{C}$. UV $\lambda_{\max }$ $(\mathrm{EtOH})\left(\log _{\mathrm{e}}\right): 225$ (4.247). ${ }^{1} \mathrm{H}-\mathrm{NMR}\left(\mathrm{DMSO}-\mathrm{d}_{6} 500 \mathrm{MHz}\right)$ $\delta$ (ppm): $1.11(3 \mathrm{H}, \mathrm{d}, \mathrm{J}=6.42,4-\mathrm{CH} 3), 1.46(1 \mathrm{H}, \mathrm{m}, \mathrm{H}-6)$, 1.48 (1H, m, H-5), 1.75 (1H, m, H-5), 1.82 (1H, m, H-6), 2.19 (1H, m, H-7), 2.28 (1H, m, H-7), 3.36 (1H, d, J = 15.57, $\mathrm{H}-1), 3.39(1 \mathrm{H}, \mathrm{d}, \mathrm{J}=15.57, \mathrm{H}-1)$, $4.74(1 \mathrm{H}, \mathrm{m}, \mathrm{H}-4), 6.23$ (2H, s, H-12, H-14), 6.3 (1H, d, J = 15.74, H-9), $6.38(1 \mathrm{H}$, m, H-8). ${ }^{13}$ C-NMR (DMSO-d $\left.6500 \mathrm{MHz}\right) \delta$ (ppm): 20.11 (q, 4- $\mathrm{CH}_{3}$ ), 23.94 (t, C-6), 32.9 (t, C- 7), 33.53 (t, C-5), 39.94 (t, C-1), 72.28 (d, C-4), 101.62 (d, C-12), 109.71 (d, C-14), 118.18 (s, C-16), 132.72 (d, C-9), 133.95 (s, C-15), 153.75 (d, C-8), 157.62 (s, C-13), 159.25 (s, C-11), 170.43 (s, C-2), 197.75 (s, C-10). IR (KBr) $\left(v_{\max } \mathrm{cm}^{-1}\right): 3470$ and 3300 (hydroxyl), 1720 (lactone), 1600 (conjugated aromatic system), 1300 and 970 (conjugated trans- $\mathrm{CH}=\mathrm{CH}-$ ), 843 (1,2,3,5-tetrasubstituted benzene). MS (ESI) $m / z$ : 291.1 [M $+\mathrm{H}]^{+}$; HR-MS (ESI) $m / z$ calculated for $\mathrm{C}_{16} \mathrm{H}_{18} \mathrm{O}_{5}[\mathrm{M}+\mathrm{H}]^{+}$: 290.32, found: 291.122. Based on the spectral data (Additional file 1: Figure S1-S7) and its comparison with that of the authentic data from the reported literature (Munro et al. 1976; Arai et al. 1989; Jiang et al. 2008), the compound 1 was identified as $\alpha, \beta$-dehydrocurvularin (Figure 1).

Compound 2: Yield: $60 \mathrm{mg} \mathrm{l}^{-1}$; white powder $(\mathrm{MeOH})$, mp 205-208 ${ }^{\circ} \mathrm{C}$. UV $\lambda_{\max }(\mathrm{EtOH})\left(\log _{\mathrm{e}}\right): 225$ (4.247). MF: $\mathrm{C}_{16} \mathrm{H}_{20} \mathrm{O}_{5} ;{ }^{1} \mathrm{H}$ NMR $\left(\mathrm{CDCl}_{3}, 300 \mathrm{MHz}\right) \delta$ (ppm): 9.49 (1 H, s, 16-OH), 9.26(1 H, s, 14-OH), 6.38 (1 H, s, H-15), 6.15 (1 H, d, H-13), 4.87 (1 H, m, H-8), 3.64 (2 H, s, H-11), 2.77 ( $1 \mathrm{H}, \mathrm{m}, \mathrm{H}-3 \mathrm{a}), 2.56$ ( $1 \mathrm{H}, \mathrm{d}, \mathrm{m}, \mathrm{H}-3 \mathrm{~b}), 1.77-1.18$ $(8 \mathrm{H}, \mathrm{m}, \mathrm{H}-4,5,6,7), 1.14(3 \mathrm{H}, \mathrm{d}, 8-\mathrm{CH} 3) .{ }^{13} \mathrm{C} \mathrm{NMR}$ data $\left(\mathrm{CDCl}_{3}, 300 \mathrm{MHz}\right) \delta$ (ppm): 206.05 (C-2), 170.19 (C-10), 159.13 (C-16), 157.28 (C-14), 135.28 (C-12), 119.62 (C-1), 110.92 (C-13), 101.59 (C-15), 71.42 (C-8), 42.89 (C-3), 40.33 (C-11), 31.56 (C-7), 26.18 (C-5), 23.28 (C-6), 22.26 (C-4), 20.08 (8-CH3). IR (KBr): 3425 (br), 2924, 1722, 1609, 1592, 1264. MS (ESI) $m / z: 293[\mathrm{M}+\mathrm{H}]^{+}$; HR-MS (ESI) $m / z$ calculated for $\mathrm{C}_{16} \mathrm{H}_{20} \mathrm{O}_{5}[\mathrm{M}+\mathrm{H}]^{+}: 292.33$, found: 292.11. The spectral data of the compound 2 (Additional file 1: Figure S8; Additional file 1: Figure S9; Additional file 1: Figure S10; Additional file 1: Figure S11; Additional file 1: Figure S12; Additional file 1: Figure S13;Additional file 1: Figure S14; Additional file 1: Figure S15; Additional file 1: Figure S16) were identical with that of the authentic data from the reported literature (Musgrave 1957; Ghisalberti et al. 1993) and was identified as curvularin (Figure 1).

\section{Acetylcholine esterase inhibitory activity}

The initial crude extract showed $60 \%$ of AChE inhibitory activity. Among the two purified compounds, only curvularin exhibited positive AChE inhibitory activity showing $80 \%$ inhibition as compared to standard galanthamine which showed $75 \%$ inhibition. The $\mathrm{IC}_{50}$ values determined for curvularin and galanthamine (standard) were 1.36 and $2.625 \mu \mathrm{M} \mathrm{ml}^{-1}$.

\section{Antimicrobial susceptibility testing and cytotoxicity}

Antimicrobial susceptibility tests of the purified metabolites profiled from strain BK-3 indicated that both the compounds did not exhibit any antimicrobial activity against all<smiles>C[C@@H]1CCCC=CC(=O)c2c(O)cc(O)cc2CC(=O)O1</smiles>

(a) $\alpha, \beta$-Dehydrocurvularin<smiles>C[C@@H]1CCCCCC(=O)c2c(O)cc(O)cc2CC(=O)O1</smiles>

(b) Curvularin

Figure 1 Structures for (a) $a, \beta$-dehydrocurvularin and (b) curvularin produced by Chrysosporium lobatum strain BK-3. 
Table 1 In vitro cytotoxicity of bioactive compounds produced by Chrysosporium lobatum strain BK-3

\begin{tabular}{lccccccc}
\hline \multicolumn{1}{c}{ Compounds } & \multicolumn{7}{c}{$\mathbf{I C}_{\mathbf{5 0}}$ values $(\boldsymbol{\mu M})\left(\right.$ Mean \pm S.D..$\left.^{\mathbf{b}}\right)$} \\
\cline { 2 - 8 } & MCF-7 & A549 & MDA-MB-231 & COLO 205 & K562 & HeLa & HEK293 (normal) \\
\hline a, B-Dehydrocurvularin & $11.19 \pm 0.31$ & $2.10 \pm 0.33$ & $9.34 \pm 0.38$ & $7.9 \pm 0.09$ & $--^{a}$ & $21.01 \pm 0.19$ & - \\
Curvularin & $21.89 \pm 0.19$ & $13.91 \pm 0.15$ & $1.3 \pm 0.37$ & - & - & $25.64 \pm 0.37$ & - \\
Doxorubicin (Standard) & $1.05 \pm 0.37$ & $1.21 \pm 0.41$ & $0.501 \pm 0.26$ & $0.985 \pm 0.33$ & $0.318 \pm 0.26$ & $0.451 \pm 0.25$ & - \\
\hline
\end{tabular}

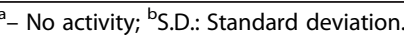

the tested Gram-positive and -negative bacteria and Candida albicans. Further, the in vitro cytotoxicity evaluated against a panel of human tumor cell lines (Table 1), indicated that both curvularin and $\alpha, \beta$-dehydrocurvularin had similar level of anti-tumour activity against A549, HeLa, MDA-MB-231 and MCF-7, while $\alpha$, $\beta$-dehydrocurvularin was active against COLO 205 with an $\mathrm{IC}_{50}$ of $7.9 \mu \mathrm{M}$; however, curvularin was inactive.

\section{Antioxidant activities}

The $\mathrm{EC}_{50}\left(\mu \mathrm{g} \mathrm{ml}^{-1}\right)$ values of the total DPPH scavenging, superoxide anion scavenging, lipid peroxidation inhibition, erythrocyte hemolytic inhibition activities of curvularin, $\alpha, \beta$-dehydrocurvularin, ascorbic acid and luteolin (as standards) is shown in Table 2. $\alpha, \beta$-Dehydrocurvularin showed significant antioxidant potential, however, curvularin did not exhibit any antioxidant activity.

\section{Molecular docking studies}

The molecular docking studies were carried out to evaluate the binding affinity of curvularin and the reversible cholinesterase inhibitor galanthamine with acetyl cholinesterase (AChE) enzyme using GOLD (version 3.2) and CDOCKER (Discovery Studio version 2.5) docking softwares. These studies were performed on curvularin and galanthamine with well-known enzyme acetylcholinesterase complexed with E2020 which is marketed as Aricept (PDBID: 1EVE) (Kryger et al. 1999). The active site radius was set to $10 \AA$, which formed the atom number 1284 , and the nitrogen atom of the active site residue Trp84, which formed $\pi-\pi$ interactions with E2020. The default parameters had been used for the docking studies in the above mentioned docking softwares. The GOLD docking results showed good activity with 49.736 and 52.06 docking scores, CDOCKER showed 53.93 and 37.72 docking scores for curvalarin and galanthamine, respectively (Table 3). The interactions of ligands in the binding site have been depicted in Figure 2.

The compound curvularin exhibited strong hydrogen bonding interactions $(\mathrm{CH}-\mathrm{O}$ and $\mathrm{OH}-\mathrm{O})$ with ASP72, GLY119 and PHE331 residues. The N-H of the HIS440 residue showed a strong hydrogen bonding interaction with oxygen of the hydroxyl group presented in the curvularin. The ligand formed $\mathrm{OH}-\mathrm{O}$ type of hydrogen bonding interactions with hydroxyl groups of tyrosine residues TYR334, 70 and 121 in the active site. The PHE330, 331 and TYR334 residues formed hydrophobic interactions with the curvularin ligand. All the above mentioned interactions were in the range of less than $4.00 \AA$ between ligand and binding site residues. The curvularin showed similar interactions as Aricept ligand, the bottom of the binding site showed a strong interaction on one face of the benzyl ring and displayed a classic $\pi-\pi$ stacking with the six-membered ring of TRP84 indole. At the middle of the binding site curvularin showed an interaction with PHE330 and TYR121 residues in the hydrophobic fashion. On other hand this ligand also formed a strong hydrogen bond interaction with HIS440 residue. The docking results of curvularin molecule were compared with the existing drug galanthamine to compare the various parameters such as score and different type of interactions. Galanthamine showed strong hydrogen bonding interactions with ASP72, GLY118 and TYR121 residues similar to curvularin, however, the strong hydrogen bonding interactions with HIS440 and GLY119 residues which was absent in galanthamine. The galanthamine showed another important hydrophobic interaction with PHE331 and TYR334 which is similar to curvularin interactions in the middle of the binding site. The above analysis clearly shows that

Table 2 Antioxidant properties of bioactive compounds produced by Chrysosporium lobatum strain BK-3

\begin{tabular}{|c|c|c|c|c|}
\hline \multirow[t]{2}{*}{ Assays } & \multicolumn{4}{|c|}{$\mathrm{EC}_{50}\left(\mu \mathrm{g} \mathrm{ml}^{-1}\right)\left(\right.$ Mean \pm S.D. $\left.{ }^{\mathrm{b}}\right)$} \\
\hline & $a, \beta$-Dehydrocurvularin & Curvularin & Ascorbic acid (Standard) & Luteolin (Standard) \\
\hline DPPH scavenging activity & $50.75 \pm 0.14$ & $-{ }^{a}$ & $40.28 \pm 0.27$ & $44.18 \pm 0.15$ \\
\hline Superoxide anion scavenging activity & $16.71 \pm 0.16$ & - & $21.01 \pm 0.36$ & $31.01 \pm 0.11$ \\
\hline Lipid peroxidation inhibition & $159.09 \pm 0.09$ & - & $139.97 \pm 0.30$ & $134.1 \pm 0.19$ \\
\hline Erythrocyte hemolytic inhibition & $141.79 \pm 0.21$ & - & $119.81 \pm 0.30$ & $122.00 \pm 0.10$ \\
\hline
\end{tabular}

a - No activity; ${ }^{\text {b}}$ S.D.: Standard deviation. 
Table 3 Docking results of curvularin produced by Chrysosporium lobatum strain BK-3 with acetyl cholinesterase enzyme (PDBID: 1EVE) using various docking protocols

\begin{tabular}{lcc}
\hline \multirow{2}{*}{ Structure } & \multicolumn{2}{c}{ Different docking protocols } \\
\cline { 2 - 3 } & GOLD & CDOCKER \\
\hline Curvularin & 44.40 & 53.93 \\
Galanthamine & 52.06 & 37.72 \\
\hline
\end{tabular}

curvularin molecule showed similar type of interactions as the existing drug galanthamine in the active site.

\section{Discussion}

AChE inhibitors have therapeutic applications in AD, senile dementia, ataxia, myasthenia gravis and Parkinson's disease (McGleenon et al. 1999). Global interests in natural products have increased of late due to safety concerns of synthetic products. All of the known AChE inhibiting drugs used in the clinics suffer from several side effects such as high toxicity, short duration of biological action, low bioavailability and narrow therapeutic effects (Lee et al. 2011). Consequently, there is a need for the development of new AChE inhibitors with less toxicity and more potency. Only three fungal species like Penicillium sp. (Kuno et al. 1996; Kim et al. 2001), Aspergillus terreus (Kim et al. 2003), and Sporothrix sp. (Wen et al. 2009) have been reported so far to produce compounds with AChE inhibitory activity. In the present study, we report the bioassay-guided purification of $\alpha$, $\beta$-dehydrocurvularin and curvularin (Figure 1) from Chrysosporium lobatum strain BK-3 and their structure elucidation. Based on the AChE inhibition and cytotoxicity screen-ing assays, we found that curvularin and $\alpha, \beta$ dehydrocurvularin have slightly different bioactivity profiles. $\alpha, \beta$-Dehydrocurvularin and curvularin and their derivatives were earlier reported to be produced by some fungi such as Aspergillus aureofulgens (Caputo and Viola 1977), Alternaria cinerariae (Arai et al. 1989), Curvularia eragrostidis (Bicalho et al. 2003), Penicillium sp. (Zhan et al. 2004), Nectria galligena (Gutierrez et al. 2005) and Eupenicillium sp. (Xie et al. 2009). Curvularin is a
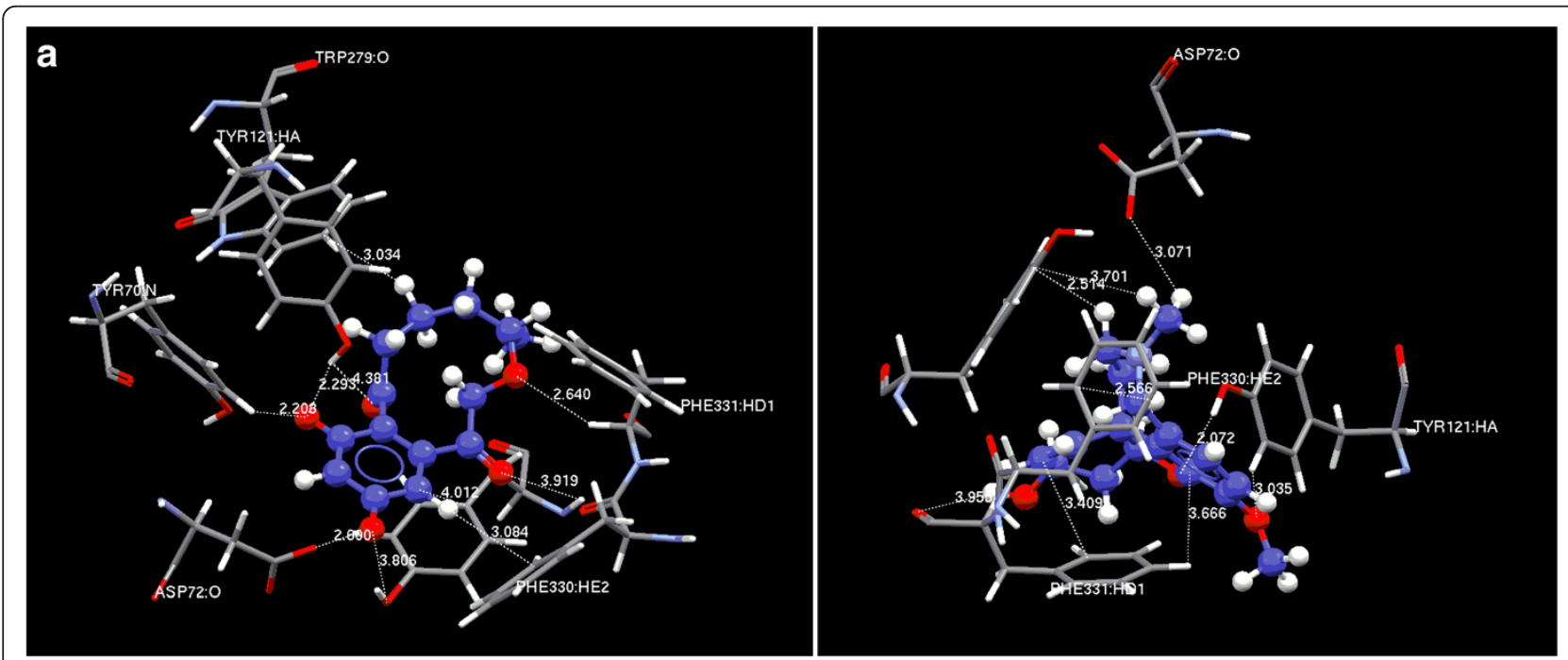

b
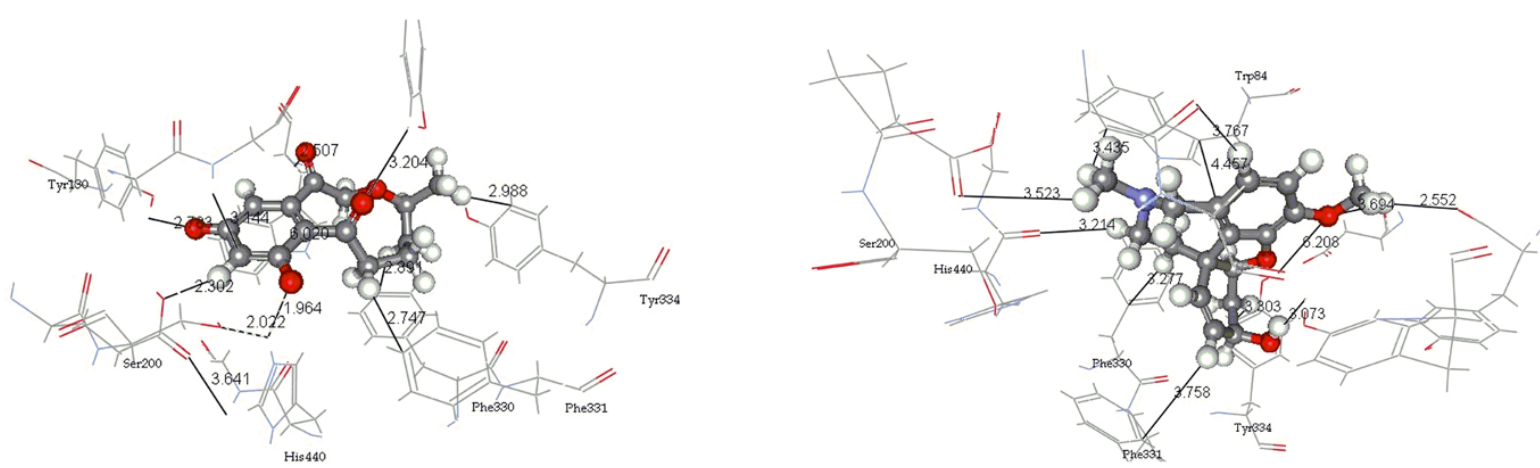

Figure 2 The typical residue interactions of curvularin and galanthamine in the active site of acetylcholinesterase enzyme (PDBID: 1EVE) using (a) GOLD and (b) CDOCKER docking protocols. 
macrolide exhibiting antibiotic activity towards some fungi and acted as a non-specific phytotoxin (Robeson and Strobel 1981). Other activities reported for curvularin and its derivatives are as potential nematicides against the rootlesion nematode, Pratylenchus penetrans (Kusano et al. 2003) and as a bioherbicide (Jiang et al. 2008). More interestingly, these derivatives were identified as cell division inhibitors (Ghisalberti et al. 1993), specifically disordering the microtubule centers (Almassi et al. 1994) and inducing barrel-shaped spindles (Kobayashi et al. 1988).

The anti-tumour activity of curvularin was not reported earlier and the $\mathrm{IC}_{50}$ values were assayed according to the MTT method. The results showed that both curvularin and $\alpha, \beta$-dehydrocurvularin were active against the tumour cell lines. The $\mathrm{IC}_{50}$ values of $\alpha$, $\beta$-dehydrocurvularin were almost always one order of magnitude higher than those of curvularin against the corresponding test cell lines except for MDA-MB-231 cell line, which might be due to the lack of the 3,4-double bond (unsaturation) in curvularin. Based on their chemical structures (Figure 1), there is only one functional group difference between the two compounds; $\alpha, \beta$-dehydrocurvularin has a double bond between $\mathrm{C}-3$ and $\mathrm{C}-4$ position, while curvularin has none in the corresponding position. In case of flavonoid-type compounds, the structure-activity relationship (SAR) was explained by the fact that the presence of a double bond is the only structural difference between two flavonoids (as one counterpart), which is one of the important structural property essential for effective interaction between flavonoid and the breast cancer resistance protein, and the flavonoid compound with double bond is more active than its counterpart (Zhang et al. 2005). It was earlier reported that the bioactivity of some other class of chemical compounds with double bond was notably stronger than those of their corresponding hydrogenated derivatives (Dizhbite et al. 2004; González-Díaz et al. 2007; Cheng et al. 2008). In one report (Gutierrez et al. 2005), it was indicated that $\alpha, \beta$-dehydrocurvularin produced by Nectria galligena showed no $\mathrm{IC}_{50}$ value reported for $\alpha$, $\beta$-dehydrocurvularin even at the use concentration of $>300 \mu \mathrm{m}$. Further, the $\alpha$, $\beta$-dehydrocurvularin exhibited cytotoxic activity with $\mathrm{IC}_{50}$ value of $<12 \mu \mathrm{g} \mathrm{ml}^{-1}$ against human lung fibroblast cell line (MRC-5), while the $\alpha, \beta$-dehydrocurvularin produced by Chrysosporium lobatum strain BK-3 showed cytotoxic activity with $\mathrm{IC}_{50}$ values of $2.10 \mu \mathrm{M}$ against A549, $7.9 \mu \mathrm{M}$ against COLO-205 and 9.34 $\mu \mathrm{M}$ against MDA-MB-231. From these results, it clears that $\alpha$, $\beta$-dehydrocurvularin from Nectria galligena exhibited only cytotoxic activity but no AChE inhibitory activity. Further, $\alpha, \beta$-dehydrocurvularin from Nectria galligena exhibited antimicrobial activity only against Pseudomonas syringae with $\mathrm{IC}_{350}$ value of $14.2 \mu \mathrm{g} \mathrm{ml}^{-1}$, while $\alpha$, $\beta$-dehydrocurvularin from Chrysosporium lobatum did not exhibit any antimicrobial activity against the tested microbial strains.

\section{Conclusions}

This study demonstrated that curvularin and $\alpha, \beta-$ dehydrocurvularin were the two main structurally related secondary metabolites produced by Chrysosporium lobatum strain BK-3 with slightly different bioactive profiles. Both these compounds were active against different cancer cell lines. However, $\alpha, \beta$-dehydrocurvularin did not exhibit inhibition for $\mathrm{AChE}$, while curvularin did, which suggested that the partial planar backbone structure in the presence of a 3,4-double bond played an important role in the inhibition of AChE activity. Further, the docking analysis showed that similar to Aricept ligand, curvularin showed a strong hydrogen bonding and hydrophobic interactions in the bottom and middle of the binding site of AChE enzyme. Curvularin had good docking scores as compared to the existing drug galanthamine; in case of CDOCKER curvularin had higher docking score than galanthamine (53.931 and 37.72, respectively). The docking study clearly revealed that the curvularin molecule had better interactions and it was fitting very well into the receptor of $\mathrm{AChE}$ enzyme. It was showing better interactions than galanthamine. To the best of our knowledge this is a first study on the characterization of metabolites of Chrysosporium lobatum strain BK-3 isolated from Kaziranga National Park biosphere exhibiting AChE inhibitory, cytotoxicity and antioxidant activities.

\section{Additional file}

Additional file 1: Figure S1. ${ }^{1} \mathrm{H}$ NMR spectrum of $\mathrm{a}, \beta$-dehydrocurvularin. Figure S2. ${ }^{13} \mathrm{C}$ NMR spectrum of $\alpha, \beta$-dehydrocurvularin. Figure S3. FT-IR spectrum of $a, \beta$-dehydrocurvularin. Figure S4. HR-MS spectrum of $a$, $\beta$-dehydrocurvularin. Figure S5a. HMBC spectrum (A) and its expansion (B) of $a, \beta$-dehydrocurvularin. Figure S5b. HMBC spectrum and its expansions ( $C$ and D) of $a, \beta$-dehydrocurvularin. Figure S6a. HSQC spectrum (A) of $a$, $\beta$-dehydrocurvularin. Figure S6b. HSQC spectrum and its expansions ( $B$ and C) of $a, \beta$-dehydrocurvularin. Figure S7. DEPT-135 spectrum of $a, \beta-$ dehydrocurvularin. Figure S8. ${ }^{1} \mathrm{H}$ NMR spectrum of curvularin. Figure S9. ${ }^{13} \mathrm{C}$ NMR spectrum of curvularin. Figure S10. FT-IR spectrum of curvularin. Figure S11. HR-MS spectrum of curvularin. Figure S12a. ${ }^{1} \mathrm{H}-{ }^{1} \mathrm{H}$ COSY spectrum (A) of curvularin. Figure S12b. ${ }^{1} \mathrm{H}-{ }^{1} \mathrm{H}$ COSY spectrum and its expansions ( $B$ and $C$ ) of curvularin. Figure S13. NOESY spectrum (A) and its expansion (B) of curvularin. Figure S14a. HMBC spectrum (A) of curvularin. Figure S14b. HMBC spectrum and its expansions ( $B$ and $C$ ) of curvularin. Figure S15a. HSQC spectrum (A) of curvularin. Figure S15b. HSQC spectrum and its expansions ( $B$ and C) of curvularin. Figure S16. DEPT-135 spectrum of curvularin.

Competing interests

The authors declare that they have no competing interests.

\section{Authors' contributions}

CGK and AK carried out the study conception and design, the analysis and interpretation of data, the drafting and revision of this manuscript. PM, PS and $J$ J carried out the acquisition of the data. GS and KSB were involved in the structural studies. KVSR performed the 2D-NMR studies. UP and GNS 
were involved in the molecular docking studies. All the authors read and approved the final manuscript.

\section{Acknowledgements}

The authors are thankful to Dr. T.C. Bora, Scientist, CSIR-North Eastern Institute of Science and Technology, Jorhat, Assam and Mr. Dharnidhar Boro, Forest Range Officer, Kaziranga National Park, Assam, for their help and providing the necessary logistics during the expedition of sample collection in the Kaziranga National Park, Assam, India. The authors acknowledge the financial assistance provided in the form of a Senior Research Fellowships to PM, GS and UP by Council of Scientific and Industrial Research (CSIR), New Delhi. The financial support extended by Council of Scientific and Industrial Research (CSIR), New Delhi, Government of India in the form of a Network Project on "Exploitation of Microbial Wealth of India" is gratefully acknowledged.

\section{Author details}

${ }^{1}$ Chemical Biology Laboratory, CSIR-Indian Institute of Chemical Technology, Uppal Road, Hyderabad 500007, Andhra Pradesh, India. ${ }^{2}$ Natural Products Chemistry Division, CSIR-Indian Institute of Chemical Technology, Uppal Road, Hyderabad 500007, Andhra Pradesh, India. ${ }^{3}$ Nuclear Magnetic Resonance Centre, CSIR-Indian Institute of Chemical Technology, Uppal Road, Hyderabad 500007, Andhra Pradesh, India. ${ }^{4}$ Molecular Modelling Group, CSIR-Indian Institute of Chemical Technology, Uppal Road, Hyderabad 500 007, Andhra Pradesh, India.

Received: 5 November 2012 Accepted: 11 March 2013 Published: 21 March 2013

\section{References}

Almassi F, Ghisalberti EL, Skelton BW, White AH (1994) Structural study of dehydrocurvularin, an inhibitor of microtubule assembly. Aust J Chem 47:1193-1197. doi:10.1071/CH9941193

Arai K, Rawlings BJ, Yoshizawa Y, Vederas JC (1989) Biosynthesis of antibiotic A26771B by Penicillium turbatum and dehydrocurvularin by Alternaria cinerariae: comparison of stereochemistry of polyketide and fatty acid enoyl thiol ester reductases. J Am Chem Soc 111:3391-3399. doi:10.1021/ ja00191a042

Badrinarayan P, Sastry GN (2011) Sequence, analysis of p38 MAP kinase: Exploiting DFG-out conformation as a strategy to design new Type II leads. J Chem Informat Modeling 51:115-129. doi:10.1021/ci100340w

Badrinarayan P, Srivani P, Sastry GN (2011) Design of 1-arylsulfamido-2alkylpiperazine derivatives as secreted PLA2 inhibitors. J Mol Modeling 17:817-831. doi:10.1007/s00894-010-0752-2

Bar-On P, Millard CB, Harel M, Dvir H, Enz A, Sussman JL, Silman I (2002) Kinetic and structural studies on the interaction of cholinesterases with the antiAlzheimer drug rivastigmine. Biochemistry 41:3555-3564. doi:10.1021/ bi020016x

Bartus RT, Dean RLR, Beer B, Lippa AS (1982) The cholinergic hypothesis of geriatric memory dysfunction. Science 217(4558):408-414. doi:10.1126/ science.7046051

Bicalho B, Goncalves RAC, Zibordi APM, Manfio GP, Marsaioli AJ (2003) Antimicrobial compounds of fungi vectored by Clusia spp. (Clusiaceae) pollinating bees. Z Naturforsch C: J Biosci 58:746-751

Caputo O, Viola F (1977) Isolation of a, $\beta$-dehydrocurvularin from Aspergillus aureofulgens. Planta Med 31:31-32

Cheng SS, Liu JY, Chang EH, Chang ST (2008) Antifungal activity of cinnamaldehyde and eugenol congeners against wood-rot fungi. Biores Technol 99:5145-5149. doi:10.1016/j.biortech.2007.09.013

Davis KL, Powchik P (1995) Tacrine. Lancet 345:625-630. doi:10.1016/S0140-6736 (95) $90526-X$

Dizhbite T, Telysheva G, Jurkjane V, Viesturs U (2004) Characterization of the radical scavenging activity of lignins - natural antioxidants. Biores Technol 95:309-317. doi:10.1016/j.biortech.2004.02.024

Ellman GL, Courtney KD, Andres V Jr, Fatherstone RM (1961) A new and rapid colorimetric determination of acetylcholinesterase activity. Biochem Pharmacol 7:88-95. doi:10.1016/0006-2952(61)90145-9

Ghisalberti EL, Hockless DCR, Rowland CY, White AH (1993) Structural study of curvularin, a cell division inhibitor. Aust J Chem 46:571-575. doi:10.1071/ CH9930571
González-Díaz H, Olazábal E, Santana L, Uriarte E, González-Díaz Y, Castañedob N (2007) QSAR study of anticoccidial activity for diverse chemical compounds: prediction and experimental assay of trans-2-(2-nitrovinyl)furan. Bioorg Med Chem 15:962-968. doi:10.1016/j.bmc.2006.10.032

Greenblatt HM, Kryger G, Lewis T, Silman I, Sussman JL (1999) Structure of acetylcholinesterase complexed with (-)-galanthamine at $2.3 \AA$ resolution. FEBS Lett 463:321-326

Gutierrez M, Theoduloz C, Rodriguez J, Lolas M, Schmeda-Hirschmann G (2005) Bioactive metabolites from the fungus Nectria galligena, the main apple canker agent in Chile. J Agric Food Chem 53:7701-7708. doi:10.1021/ jf051021।

Harvey A (2000) Strategies for discovering drugs from previously unexplored natural products. Drug Discov Today 5:294-300. doi:10.1016/S1359-6446(00) 01511-7

Höller U, Wright AD, Matthee GF, Konig GM, Draeger S, Aust HJ, Schulz B (2000) Fungi from marine sponges: diversity, biological activity and secondary metabolites. Mycol Res 104:1354-1365. doi:10.1017/S0953756200003117

Jiang S-J, Qiang S, Zhu Y-Z, Dong Y-F (2008) Isolation and phytotoxicity of a metabolite from Curvularia eragrostidis and characterisation of its modes of action. Annals Appl Biol 152:103-111. doi:10.1111/j.1744-7348.2007.00202.x

Johnson N, Davis T, Bosanquet N (2000) The epidemic of Alzheimer's disease; how can we manage the costs? PharmacoEconomics 18:215-223. doi:10.2165/00019053-200018030-00002

Kawakami Y, Inoue A, Kawai T, Wakita M, Sugimoto H, Hopfinger AJ (1996) The rationale for E2020 as a potent acetylcholinesterase inhibitor. Bioorg Med Chem Lett 4:1429-1446. doi:10.1016/0968-0896(96)00137-X

Kim W-G, Song N-K, Yoo I-D (2001) Quinolactacins A1 and A2, new acetylcholinesterase inhibitors from Penicillium citrinum. J Antibiot (Tokyo) 54:831-835. doi:10.7164/antibiotics.54.831

Kim W-G, Cho K-M, Lee C-K, Yoo I-D (2003) Terreulactones A, B, C, and D: novel acetylcholinesterase inhibitors produced by Aspergillus terreus. J Antibiot (Tokyo) 56:351-357. doi:10.7164/antibiotics.56.351

Kobayashi A, Hino T, Yata S, Itoh TJ, Sato H, Kawazu K (1988) Unique spindle poisons, curvularin and its derivatives, isolated from Penicillium species. Agric Biol Chem 52:3119-3123. doi:10.1271/bbb1961.52.3119

Kryger G, Silman I, Sussman JL (1999) Structure of acetylcholinesterase complexed with E2020 (Aricept): implications for the design of new antiAlzheimer drugs. Structure 7:297-307. doi:10.1016/S0969-2126(99)80040-9

Kumar CG, Mamidyala SK (2011) Extracellular synthesis of silver nanoparticles using culture supernatant of Pseudomonas aeruginosa. Coll Surf B Biointerf 84:462-466. doi:10.1016/j.colsurfb.2011.01.042

Kumar CG, Mongolla P, Joseph J, Nageswar YVD, Kamal A (2010) Antimicrobial activity from the extracts of fungal isolates of soil and dung samples from Kaziranga National Park, Assam, India. J Mycologie Medicale 20:283-289. doi:10.1016/j.mycmed.2010.08.002

Kumar CG, Mongolla P, Pombala S, Kamle A, Joseph J (2011) Physicochemical characterization and antioxidant activity of melanin from a novel strain of Aspergillus bridgeri ICTF-201. Lett Appl Microbiol 53:350-358. doi:10.1111/ j.1472-765X.2011.03116.x

Kuno F, Otoguro K, Shiomi K, Iwai Y, Omura S (1996) Arisugacin A and B, novel and selective acetylcholinesterase inhibitors from Penicillium sp. FO 4259. I. Screening, taxonomy, fermentation, isolation and biological activity. J Antibiot (Tokyo) 49:742-747

Kusano M, Nakagami K, Fujioka S, Kawano T, Shimada A, Kimura Y (2003) $\beta$, $\gamma$-dehydrocurvularin and related compounds as nematicides of Pratylenchus penetrans from the fungus Aspergillus sp. Biosci Biotechnol Biochem 67:1413-1416. doi:10.1.1271/bbb.67.1413

Lee S-H, Sancheti SA, Bafna MR, Sancheti SS, Seo S-Y (2011) Acetylcholinesterase inhibitory and antioxidant properties of Rhododendron yedoense var. poukhanese bark. J Med Plant Res 5:248-254

Lin Y, Wu X, Feng S, Jiang G, Luo J, Zhou S, Vrijmoed LLP, Jones EBG, Krohn K, Steongröver K, Zsila F (2001) Five unique compounds: xyloketales from mangrove fungus Xylaria sp. from the South China Sea coast. J Org Chem 66:6252-6256. doi:10.1021/jo015522r

Liu F, Ooi VE, Chang ST (1997) Free radical scavenging activities of mushroom polysaccharide extracts. Life Sci 60:763-771. doi:10.1016/S0024-3205(97) 00004-0

McGleenon BM, Dynan KB, Passmore AP (1999) Acetylcholinesterase inhibitors in Alzheimer's disease. Br J Clin Pharmacol 48:471-480. doi:10.1046/j.13652125.1999.00026.x 
Mosmann T (1983) Rapid colorimetric assay for cellular growth and survival: application to proliferation and cytotoxicity assays. J Immunol Methods 65:55-63, doi:10.1016/0022-1759(83)90303-4

Munro HD, Musgrave OC, Templeton R (1976) Curvularin. Part V. The compound $\mathrm{C}_{16} \mathrm{H}_{18} \mathrm{O}_{5}, a, \beta$-dehydrocurvularin. J Chem Soc C 10:947-948

Musgrave OC (1957) 218. Curvularin. 2. The constitution of an aromatic degradation product and the partial structure of curvularin. J Chem Soc 3:1104-1108. doi:10.1039/jr9570001104

Ng TB, Liu F, Wang ZT (2000) Antioxidative activity of natural products from plants. Life Sci 66:709-723. doi:10.1016/50024-3205(99)00642-6

Nightingale SL (1997) Donepezil approved for treatment of Alzheimer's disease. J Am Med Assoc 277:10-10

Ravindra GK, Achaiah G, Sastry GN (2008) Molecular modeling studies of phenoxypyrimidinyl imidazoles as p38 kinase inhibitors using QSAR and docking. Eur J Med Chem 43:830-838. doi:10.1016/j.ejmech.2007.06.009

Rhee IK, van de Meent M, Ingkaninan K, Verpoorte R (2001) Screening for acetylcholinesterase inhibitors from Amaryllidaceae using silica gel thin-layer chromatography in combination with bioactivity staining. J Chromatogr A 915:217-223, http://dx.doi.org/10.1016/50021-9673(01)00624-0

Robeson DJ, Strobel GA (1981) a, $\beta$-Dehydrocurvularin and curvularin from Alternaria cinerariae. Z Naturforsch C J Biosci 36:1081-1083

Srivani P, Srinivas E, Raghu R, Sastry GN (2007) Molecular modeling studies of pyridopurinone derivatives - Potential phosphodiesterase 5 inhibitors. J Mol Graphics Modeling 26:378-390. doi:10.1016/j.jmgm.2007.01.007

Strobel GA (2000) Microbial gifts from rain forests. Can J Plant Pathol 24:14-20

Tariot P (2001) Current status and new developments with galantamine in the treatment of Alzheimer's disease. Expert Opin Pharmacother 2:2027-2049. doi:10.1517/14656566.2.12.2027

Tulp M, Bohlin L (2004) Unconventional natural sources for future drug discovery. Drug Discov Today 9:450-458. doi:10.1016/S1359-6446(04)03066-1

UNEP (2005) United Nations Environment Programme. Kaziranga National Park, Assam, India. Available at http://assamforest.in/knp-osc/pdfreport/ unep_wcmc_kaziranga.pdf (Accessed 28 May 2012)

UNESCO (2010) State of conservation of world heritage properties in the AsiaPacific region - Kaziranga National Park. Available at http://whc.unesco.org/ archive/ periodicreporting/APA/cycle01/section2/337-summary.pdf (Accessed 28 May 2010)

Watkins PB, Zimmerman HJ, Knapp MJ, Gracon SI, Lewis KW (1994) Hepatotoxic effects of tacrine administration in patients with Alzheimer's disease. J Am Med Assoc 271:992-998. doi:10.1001/jama.271.13.992

Wen L, Cai X, Xu F, She Z, Chan WL, Vrijmoed LLP, Jones EBG, Lin Y (2009) Three metabolites from the mangrove endophytic fungus Sporothrix sp. (\#4335) from the South China Sea. J Org Chem 74:1093-1098. doi:10.1021/jo802096q

Xie LW, Ouyang YC, Zou K, Wang GH, Chen MJ, Sun HM, Dai SK, Li X (2009) Isolation and difference in anti-Staphylococcus aureus bioactivity of curvularin derivatives from fungus Eupenicillium sp. Appl Biochem Biotechnol 159:284-293. doi:10.1007/s12010-009-8591-2

Zeng F, Jiang H, Zhai Y, Zhang H, Chen K, Ji R (1999) Synthesis and acetylcholinesterase inhibitory activity of huperzine A - E2020 combined compound. Bioorg Med Chem Lett 9:3279-3284. doi:10.1016/50960-894X(99) 00597-1

Zhan JX, Wijeratne EMK, Seliga CJ, Zhang J, Pierson EE, Pierson LS III, Vanetten HD, Guntalika AAL (2004) A new anthraquinone and cytotoxic curvularins of a Penicillium sp. from the rhizosphere of Fallugia paradoxa of the Sonoran desert. J Antibiot (Tokyo) 57:341-344

Zhang EX, Yu L (1997) Studies on polysaccharide from Sargassum thunberg II for its ability to scavenge active oxygen species. Chinese J Marine Drugs 3:1-4

Zhang SZ, Yang XN, Coburn RA, Morris ME (2005) Structure activity relationships and quantitative structure activity relationships for the flavonoid-mediated inhibition of breast cancer resistance protein. Biochem Pharmacol 70:627-639. doi:10.1016/j.bcp.2005.05.017

\section{doi:10.1186/2193-1801-2-122}

Cite this article as: Kumar et al:: Metabolite profiling and biological activities of bioactive compounds produced by Chrysosporium lobatum strain BK-3 isolated from Kaziranga National Park, Assam, India. SpringerPlus 2013 2:122.

\section{Submit your manuscript to a SpringerOpen ${ }^{\circ}$ journal and benefit from:}

- Convenient online submission

- Rigorous peer review

- Immediate publication on acceptance

- Open access: articles freely available online

- High visibility within the field

- Retaining the copyright to your article

Submit your next manuscript at $>$ springeropen.com 